Check for updates

Cite this: J. Mater. Chem. A, 2019, 7, 2422

DOI: $10.1039 / \mathrm{c} 9 \operatorname{ta} 90018 \mathrm{~g}$

www.rsc.org/MaterialsA

\section{Correction: Pitaya-like microspheres derived from Prussian blue analogues as ultralong-life anodes for lithium storage}

\author{
Lianbo Ma, ${ }^{a}$ Tao Chen, ${ }^{a}$ Guoyin Zhu, ${ }^{a}$ Yi Hu, ${ }^{a}$ Hongling Lu, ${ }^{a}$ Renpeng Chen, ${ }^{a}$ \\ Jia Liang, ${ }^{a}$ Zuoxiu Tie, ${ }^{a}$ Zhong Jin*a and Jie Liu*ab
}

Correction for 'Pitaya-like microspheres derived from Prussian blue analogues as ultralong-life anodes for lithium storage' by Lianbo Ma et al., J. Mater. Chem. A, 2016, 4, 15041-15048.

The authors regret that the elemental mapping results shown in Fig. $1 \mathrm{i}$ and Fig. $1 \mathrm{j}$ of the published article (referring to the elemental distributions of $\mathrm{Co}_{3} \mathrm{ZnC} / \mathrm{C}$ ) were incorrect; these may have been mixed with those for other samples during the revision stage of this paper. To avoid possible misunderstanding, this sample has been prepared and characterized once again by the same methods. The corrected version of Fig. 1 (with panels (i) and (j) updated) is shown below.

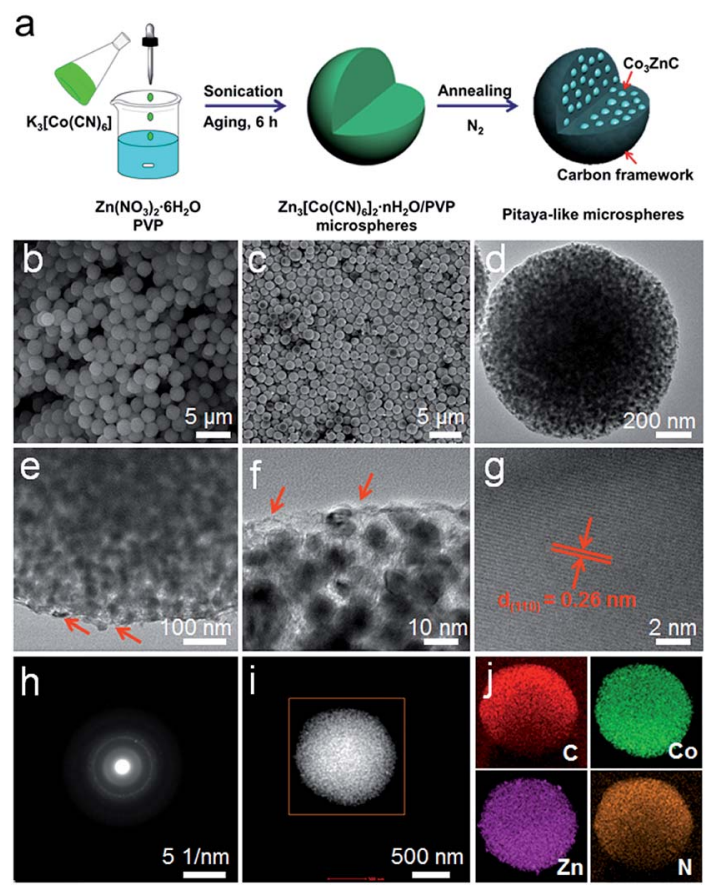

Fig. 1 Synthesis and morphology characterizations of pitaya-like microspheres. (a) Schematic illustration of the formation process of pitaya-like microspheres. (b) FESEM image of $\mathrm{Zn}_{3}\left[\mathrm{Co}(\mathrm{CN})_{6}\right]_{2} \cdot n \mathrm{H}_{2} \mathrm{O} / \mathrm{PVP}$ precursor microspheres. (c) FESEM, (d-f) TEM, (g) high-resolution TEM and (h) SAED images of as-synthesized pitaya-like microspheres, respectively. The rough edges of carbon outer-shells in (d) and (e) are marked by red arrows. The lattice distance in (g) is measured to be $0.26 \mathrm{~nm}$, corresponding to the (110) planes of the cubic phase Co $Z$ ZnC. (i) Energy-filtered TEM image of a pitaya-like microsphere and (j) the C, Co, Zn and $\mathrm{N}$ element mapping collected in the same rectangle area of (i), respectively.

The Royal Society of Chemistry apologises for these errors and any consequent inconvenience to authors and readers.

${ }^{a}$ Key Laboratory of Mesoscopic Chemistry of MOE, Collaborative Innovation Center of Chemistry for Life Sciences, School of Chemistry and Chemical Engineering, Nanjing University, Nanjing, 210093, China. E-mail: zhongjin@nju.edu.cn

${ }^{b}$ Department of Chemistry, Duke University, Durham, North Carolina, 27708, USA. E-mail: j.liu@duke.edu 